\title{
Um Framework para Avaliação de Transparência em Portais de Ecossistemas de Software a partir da Experiência de Desenvolvedor
}

\section{Rodrigo Oliveira Zacarias ${ }^{1}$ (Doutorando), Rodrigo Pereira dos Santos $^{1}$ (Orientador)}

${ }^{1}$ Doutorado em Informática

Programa de Pós-Graduação em Informática (PPGI)

Universidade Federal do Estado do Rio de Janeiro (UNIRIO)

Avenida Pasteur, 458 - CEP 22290-250 - Rio de Janeiro - RJ - Brasil

Ingresso: 03/2021 - Qualificação: 12/2022 - Previsão Defesa: 02/2025

rodrigo.zacariasdedu.unirio.br, rpseuniriotec.br

\begin{abstract}
Resumo. As interfaces que amparam a Web social (e.g., portais Web, fóruns, redes sociais etc.) desempenham um papel importante dentro de um ecossistema de software (ECOS), tendo em vista que facilitam o acesso à informação e a interação entre os desenvolvedores externos à sua plataforma tecnológica comum. Essas interfaces formam os chamados portais de ECOS. Nesse cenário, o conceito de transparência permite que os desenvolvedores acessem, entendam e aprendam os elementos e processos no contexto de desenvolvimento colaborativo. A ausência de transparência nos portais de ECOS pode trazer problemas tanto na comunicação entre os desenvolvedores como no entendimento das informações fornecidas. Isso afeta a Experiência de Desenvolvedor (DX, do inglês Developer Experience) no ECOS, podendo trazer como consequência a falta de engajamento entre os desenvolvedores externos na plataforma. Em casos mais graves, esse fato pode culminar na "morte" do ECOS. Nesse contexto, o objetivo desta pesquisa é desenvolver um framework para a avaliação da transparência em portais de ECOS a partir da DX. A base desse framework será constituída pela aplicação de heurísticas elou metaheurísticas para avaliar a transparência, que serão elaboradas ao longo deste trabalho. Para isso, é proposta uma abordagem metodológica composta de revisões de literatura, experimentos com desenvolvedores para monitoramento da DX e avaliação com especialistas. Esta pesquisa almeja como contribuições a criação do framework, para ser utilizado por profissionais de organizações que formam um ECOS ou por pesquisadores da área, o entendimento de como a transparência nos portais de ECOS afeta a DX e um conjunto de heurísticas elou metaheurísticas para transparência em portais de ECOS.
\end{abstract}

Palavras-chave. Transparência, Ecossistema de Software, Experiência de Desenvolvedor, Heurísticas.

Evento CBSoft: SBES. 


\section{Caracterização do Problema}

Com os avanços nas abordagens de desenvolvimento de software para atender às novas demandas do mercado, tem sido um desafio para as corporações manter uma arquitetura de software totalmente internalizada à organização. Por esse motivo, algumas empresas têm investido na abertura de suas arquiteturas para permitir a colaboração de desenvolvedores externos na produção de seus componentes em torno de uma plataforma tecnológica comum. Essa prática define o conceito de Ecossistema de Software (ECOS) [Santos 2016].

Nesse cenário, as interfaces que amparam a Web social (e.g., portais Web, fóruns, redes sociais etc.) desempenham um papel importante dentro do ECOS, pois facilitam o acesso à informação e a interação entre os desenvolvedores na plataforma. Essas interfaces formam os chamados portais de ECOS [Meireles et al. 2019]. Como exemplos, é possível citar os portais de ECOS móvel Android ${ }^{1}$ e $\mathrm{iOS}^{2}$ que disponibilizam para a sua comunidade de desenvolvedores um cojunto de ferramentas, documentos e canais de suporte para auxiliá-los na construção de aplicações utilizando suas respectivas tecnologias.

Os portais de ECOS são elementos fundamentais para que a organização central, proprietária da plataforma, mantenha seu ecossistema sustentável no mercado ao longo do tempo. Para que isso ocorra de forma efetiva, os atores precisam ter conhecimento dos processos e elementos (e.g., arquivos de documentação, código-fonte, fóruns etc.) que compõem a plataforma [Cataldo e Herbsleb 2010]. Neste ponto, a transparência do portal de ECOS é imprescindível [Santos et al. 2016], pois permite que os atores acessem as informações da plataforma e aprendam como utilizá-la.

Por ser um elemento crítico em um ambiente colaborativo, a ausência de transparência pode prejudicar a comunicação entre os colaboradores e dificultar o entendimento das informações disponibilizadas [Meireles et al. 2019]. Isso afeta a Experiência de Desenvolvedor (DX, do inglês Developer Experience) no ambiente, podendo trazer como consequências a falta de interesse e de engajamento entre os desenvolvedores externos à plataforma. Em casos mais graves, isso pode culminar na "morte" do ECOS [Fontão et al. 2020].

Nesse sentido, manter o engajamento dos desenvolvedores é um dos elementos críticos para a saúde do ECOS, i.e., a capacidade de um ecossistema manter produtividade, robustez e criação de nicho ao longo do tempo [Amorim et al. 2017]. Assim, a transparência é um requisito que impacta na coordenação dos desenvolvedores em um ambiente de desenvolvimento distribuído e colaborativo como o ECOS.

A disponibilidade da informação permite que os envolvidos estejam conscientes da evolução e das atividades de desenvolvimento de software. Para isso, os interessados em um ECOS devem definir o nível de transparência que desejam ter no ambiente, ou seja, quais elementos do ECOS devem estar disponíveis para eles [Santos et al. 2016]. A organização central, ciente dessas demandas, deve preparar um ambiente propício para a interação dos desenvolvedores externos. Nesse cenário, um conjunto de heurísticas e/ou metaheurísticas (i.e., conjunto de regras ou técnicas que, em geral, melhoram o desempenho de métodos de solução de problemas [Bader et al. 2017]) definidas pode auxiliar a organização central no monitoramento desse requisito.

\footnotetext{
${ }^{1}$ https://developer.android.com/

${ }^{2}$ https://developer.apple.com/
} 
A transparência nos portais contribui para a sustentabilidade e longevidade do ECOS no mercado, visto que mantém os desenvolvedores ativos em seu ambiente por mais tempo. Assim, a detecção de inconsistências dessa característica por meio do monitoramento da DX pode contribuir para a elaboração de frameworks, métodos e heurísticas que podem permitir a implementação de melhorias pela organização central em seus portais, minimizando a ocorrência de problemas futuros.

Nesse contexto, o objetivo desta pesquisa é desenvolver um framework para a avaliação da transparência em portais de ECOS a partir da DX. A base desse framework será constituída pela aplicação de heurísticas e/ou metaheurísticas para avaliar a transparência, que serão elaboradas ao longo deste trabalho. Para isso, é proposta uma abordagem metodológica composta de revisões de literatura, experimentos com desenvolvedores para monitoramento da DX e avaliação com especialistas.

Este artigo está organizado da seguinte forma: a Seção 2 traz a fundamentação teórica e, na Seção 3, são apresentadas as informações sobre a proposta do framework. A Seção 4 descreve a metodologia, seguida da comparação com os trabalhos relacionados na Seção 5. Por fim, na Seção 6, são apresentadas as contribuições esperadas.

\section{Fundamentação Teórica}

\subsection{Ecossistemas de Software}

Um ECOS pode ser definido como um conjunto de atores que funcionam como uma unidade e suas relações e interações com um mercado distribuído entre software e serviços. Essas relações são, em grande parte, centradas em uma plataforma tecnológica ou em um mercado comum, que permite a troca de informações, recursos e artefatos [Jansen et al. 2009]. Esses elementos reunidos formam um ECOS e requerem a integração de mecanismos e ferramentas de apoio para efetuarem as trocas mencionadas, com a garantia da comunicação e interação dos desenvolvedores e usuários [Santos 2016].

Nesse contexto de múltiplos atores, podem ser apontados três papeis principais: organização central, usuários finais e desenvolvedores externos. A organização central (keystone) é a organização ou grupo que conduz o desenvolvimento da tecnologia da plataforma. Os usuários finais (end-users) são clientes que precisam da plataforma para realizar seu negócio. Por fim, os desenvolvedores externos (third-parties) utilizam a plataforma como base para desenvolver novos produtos e soluções [Hanssen e Dybå 2012].

Segundo Manikas (2016), os ECOS podem ser classificados em três tipos: fechados, abertos e híbridos. Os fechados têm sua criação de valor baseada em contribuições proprietárias (e.g., SAP e Amazon). Os abertos permitem as contribuições de diferentes atores e do público (e.g., Eclipse e Apache). Por fim, os híbridos suportam contribuições proprietárias e de código aberto (e.g. Android e iOS).

\subsection{Transparência e Experiência de Desenvolvedor}

A transparência pode ser estabelecida como uma condição para que as informações referentes a capacidades, prioridades e comportamento estejam disponíveis [Lord 2007]. Grande parte das informações provenientes de processos organizacionais são geradas por meio do uso de software. Isso faz com que o conceito de transparência seja estendido ao software e seus processos de desenvolvimento [Santos et al. 2016] e, consequentemente, gera impactos para a DX. 
Cappelli (2009) ressalta um conjunto de características inerentes a um ambiente transparente e que devem ser previamente definidas e implementadas nos portais Web, as quais também podem ser associadas aos portais de ECOS: (i) Acessibilidade - capacidade de ser utilizado em diversos ambientes e por qualquer indivíduo que necessite; (ii) Usabilidade - capacidade de uso sem apresentar dificuldades ou obstáculos; (iii) Informativo - capacidade de prover informações com qualidade; (iv) Entendimento - capacidade de ter os seus processos e informações entendidos; e (v) Auditabilidade - capacidade de ser dirigido, fiscalizado e orientado [Leite e Cappelli 2010].

Antes de relacionar a transparência e DX, é preciso entender que a DX consiste nas experiências relacionadas a todos os tipos de atividades e artefatos que um desenvolvedor possa ter contato durante sua participação no projeto de um software. Ela pode ser analisada por meio das seguintes dimensões [Fagerholm e Münch 2012]: (i) dimensão cognitiva: consiste em fatores que afetam como os desenvolvedores percebem sua infraestrutura de desenvolvimento em um nível intelectual; (ii) dimensão afetiva: consiste em fatores que influenciam a maneira como os desenvolvedores se sentem sobre seu trabalho; e (iii) dimensão conativa: consiste em fatores que afetam como os desenvolvedores veem o valor de suas contribuições.

É possível visualizar a relação das características de transparência a partir da descrição dos fatores associados às dimensões de DX no contexto dos portais de ECOS. Na dimensão cognitiva, a acessibilidade e a usabilidade melhoram a interação com as ferramentas e os processos disponibilizados pela plataforma tecnológica aos desenvolvedores. Na dimensão afetiva, a usabilidade da interface e a apresentação de conteúdos informativos nos canais de suporte e os meios de comunicação da plataforma (fóruns ou mídias sociais) podem aumentar a confiança, a credibilidade e, consequentemente, as chances de fidelização de desenvolvedores. Por fim, na dimensão conativa, investir em processos auditáveis para monitorar as contribuições de desenvolvedores que chegam à plataforma pode transmitir maior clareza no valor do trabalho de quem se relaciona com o ECOS.

\section{Proposta do Framework para Avaliação de Transparência em Portais de Ecossistemas de Software a partir da Experiência de Desenvolvedor}

A transparência é um elemento-chave para coordenação, comunicação e colaboração entre os atores de ECOS. Os portais de ECOS são interfaces importantes para acesso à informação e são a principal entrada para desenvolvedores externos ao ECOS. A forma como os portais são apresentados tem grande influência na experiência e na permanência desses profissionais.

A partir disso, a proposta desta pesquisa é desenvolver um framework para sistematizar a avaliação de transparência em portais de ECOS. Para isso, serão elaboradas heurísticas e/ou metaheurísticas para avaliar cada uma das cinco características de transparência (acessibilidade, usabilidade, informativo, entendimento e auditabilidade) por meio de experimentos com o monitoramento de DX. A Figura 1 ilustra um esquema inicial do framework.

O framework será composto por três fases: (1) Coleta de Informações, (2) Avaliação e (3) Resultados. Na Fase 1, serão coletadas informações relativas ao portal de ECOS a ser analisado. O contexto de negócio é importante para se entender de 


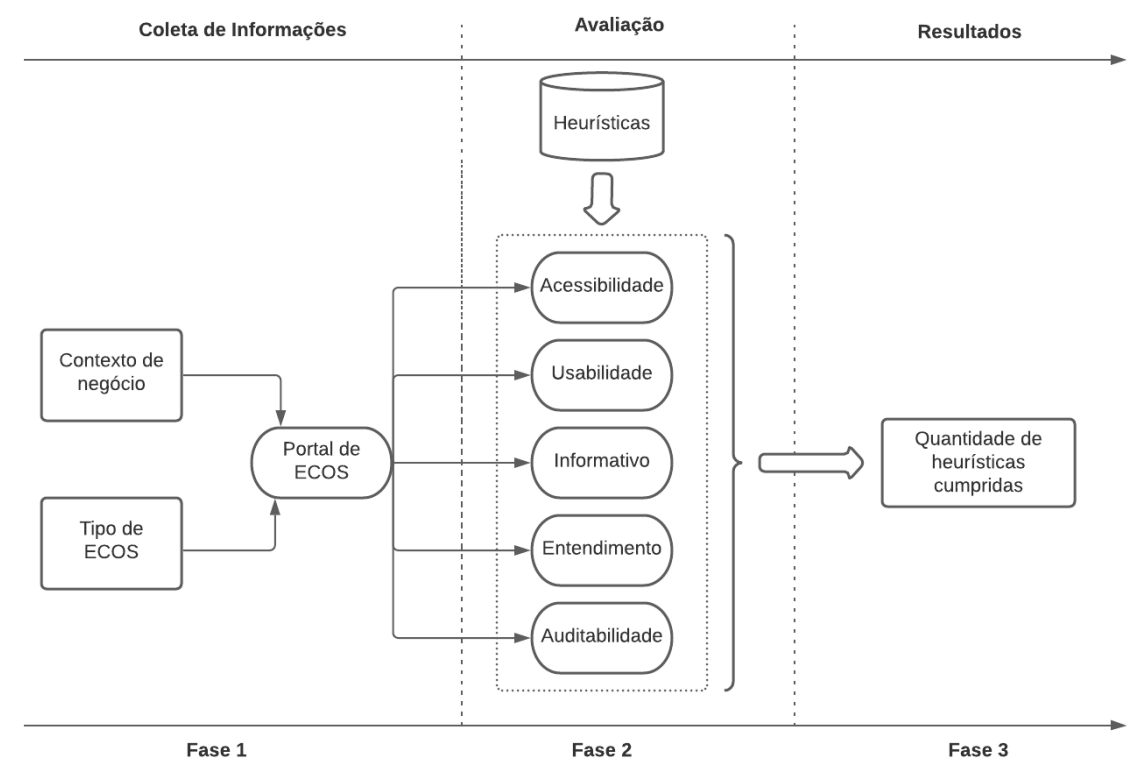

Figura 1. Esquema inicial do framework proposto

que modo a colaboração de desenvolvedores externos agrega o valor ao negócio e quais informações devem estar visíveis para tal. O tipo de ECOS também deve ser levado em consideração, tendo em vista que alguns ECOS podem apresentar restrições de acesso a informações, como os proprietários. Esses dados serão utilizados para a seleção de um subconjunto de heurísticas mais voltado ao cenário informado.

$\mathrm{Na}$ Fase 2, serão aplicadas as heurísticas e/ou metaheurísticas elaboradas previamentes por meio de revisões da literatura, experimentos com desenvolvedores e avaliação com especialistas. Nesse estágio, são verificadas quais heurísticas foram cumpridas no portal de ECOS em cada característica de transparência, considerando o contexto do negócio e o tipo de ECOS.

A Fase 3 abrangerá a análise dos resultados e a provisão do diagnóstico do nível de transparência do portal. Será analisado o quantitativo de heurísticas cumpridas e sumarizado quais os pontos de insuficiência, caso existam, bem como recomendações para minimizá-los. A ideia é que os resultados sejam utilizados por profissionais da organização central que são responsáveis pelo design dos portais. Além de utilizar as heurísticas para a avaliação, eles poderão utilizá-las como guia para a construção de novos portais de ECOS.

\section{Metodologia}

Esta pesquisa busca responder às seguintes questões de pesquisa: (1) "Como a transparência nos portais de ECOS afeta a DX?" e (2) "Como verificar o nível de transparência adequado de um portal de ECOS a partir da DX?"

Para responder essas questões que guiarão a construção do framework e a elaboração de heurísticas de transparência a partir de DX, este trabalho propõe a adoção de uma metodologia que combina diferentes métodos de pesquisa. Ela é uma adaptação da metodologia de Quiñones et al. (2018), que propõe uma sequência de atividades para a elaboração de heurísticas de usabilidade/experiência de usuário, tais como revisão de literatura, experimentos com o usuário, aplicação de questionários, validação com especialistas, refinamento e documentação de resultados. 
Assim, a trajetória de pesquisa é constituída de três etapas: (1) Estudos exploratórios, (2) Elaboração das heurísticas e/ou metaheurísticas de transparência e (3) Construção e avaliação do framework. A Figura 2 ilustra as macroatividades de cada etapa da metodologia de pesquisa.

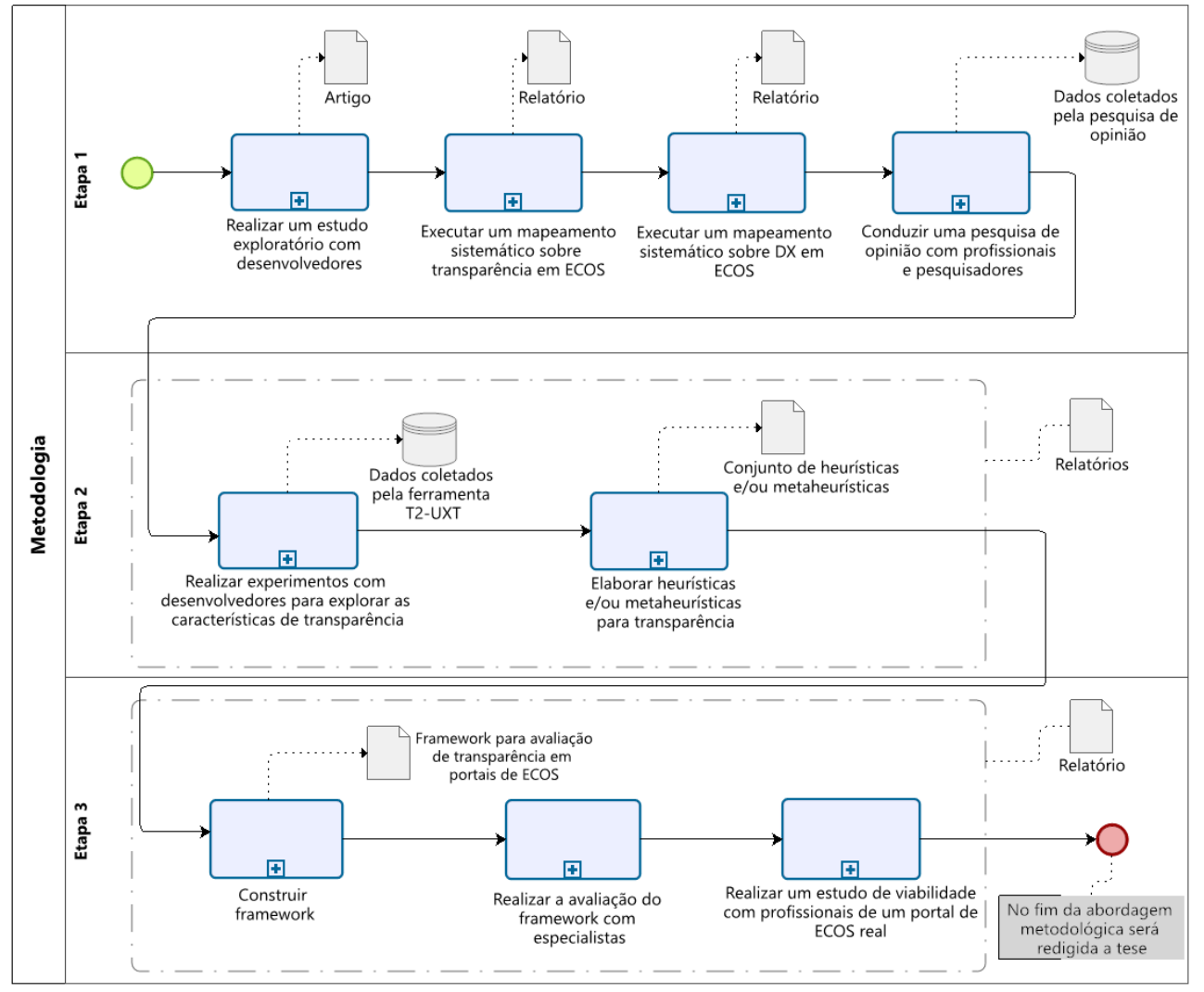

Figura 2. Etapas da metodologia de pesquisa.

Atualmente, a pesquisa está na Etapa 1, que é constituída de um experimento com desenvolvedores (já realizado) e revisões de literatura, ambos de caráter exploratório. $\mathrm{O}$ objetivo do experimento foi avaliar, de forma inicial, a relação de transparência com a DX em dois portais de ECOS híbridos: Android e iOS. Para facilitar a coleta e análise da interação dos desenvolvedores, foi utilizada a ferramenta de captura multimodal (captura do movimento do olho, mouse e dados de entrada pelo teclado) T2-UXT ${ }^{3}$ para processamento, visualização e avaliação de dados.

No caso das revisões de literatura, estão sendo preparados dois mapeamentos sistemáticos de literatura. O primeiro visa identificar como o requisito de transparência é tratado em ECOS. O segundo busca elencar fatores de DX no contexto de ECOS. Com bases nos resultados, será realizada uma pesquisa de opinião com profissionais e pesquisadores para estabelecer relações entre transparência e DX em ECOS.

Na Etapa 2, a partir dos resultados formalizados na etapa anterior, são previstos experimentos com desenvolvedores para explorar, individualmente, cada característica de transparência em diferentes portais de ECOS. A ferramenta T2-UXT também será utilizada para monitorar a DX, quando possível. A captura multimodal da interação permite

\footnotetext{
${ }^{3}$ Tracking Techniques - User eXperience Evaluation Tool [Souza et al. 2020]
} 
documentar quais pontos da interface podem ocasionar algum problema de interação e resultar em uma experiência insatisfatória, e.g., o desenvolvedor não consegue encontrar o link de acesso à documentação da plataforma na interface ou o desenvolvedor passa muito tempo focado em um trecho de texto, o que pode ser um indício de problemas de entendimento do conteúdo apresentado. O intuito é entender qual característica de transparência não está totalmente presente no portal e como isso afeta uma ou mais dimensões de DX, como apresentado na Seção 2.2.

Em conjunto com a T2-UXT, também poderá ser utilizado o Think Aloud Protocol (i.e., um método de coleta de dados onde os participantes pensam em voz alta enquanto executam tarefas específicas, permitindo ter uma visão sobre os seus processos cognitivos [Fonteyn et al. 1993]) como mais uma alternativa para a captura de informações da interação do desenvolvedor em um portal de ECOS. A partir da análise dos artefatos gerados pela ferramenta, serão elaboradas as heurísticas e/ou metaheurísticas para cada uma das características. Essas heurísticas e/ou metaheurísticas passarão por avaliação com especialistas e serão refinadas antes de serem inseridas no framework, seguindo as diretrizes de Quiñones et al. (2018).

Na Etapa 3, será construído e formalizado o framework proposto. Em um primeiro momento, o artefato terá o seu desempenho avaliado por especialistas (pesquisadores e/ou profissionais) nas áreas de DX, transparência e ECOS. Em seguida, será planejada a execução de um estudo de viabilidade utilizando o framework com profissionais que atuam no desenvolvimento do portal de um ECOS real, a fim de verificar a eficácia das heuristícas/metaheurísticas na avaliação de transparência e sua utilidade para a indústria.

Em cada etapa, é prevista a publicacação dos resultados em formatos de artigos em congressos, simpósios, periódicos e demais canais de comunicação científica com as comunidades de ECOS, Interação Humano-Computador, Engenharia de Software e Sistemas de Informação. Ao final, será redigida a tese.

\section{Trabalhos Relacionados}

Fontão et al. (2017) ressaltam a importância de se investigar a DX em um ECOS, móvel nesse caso, uma vez que permite que a organização central planeje mecanismos para manter ações de desenvolvedores que afetam os indicadores de produtividade, robustez e criação de nicho. Assim, os autores identificaram 20 fatores de influência relacionados a DX por meio de uma revisão de literatura. No trabalho de Herbsleb et al. (2016), é realizada uma discussão de que um ECOS tem que ser transparente, mas de forma inteligente. Nesse caso, os desenvolvedores precisam ter acesso somente às informações necessárias para uma melhor produtividade e experiência em ECOS.

Meireles et al. (2019) propõem um instrumento para avaliação de transparência em portais de ECOS, chamado ITRANSPE. Constitui-se em uma planilha eletrônica que contém um questionário com o objetivo de avaliar as características de transparência. Os desenvolvedores analisam se as questões se aplicam totalmente, parcialmente ou não se aplicam aos portais avaliados. Caso a avaliação aponte a insuficiência na aplicação de alguma questão, o ITRANSPE sugere algumas medidas corretivas que podem ser implementadas naquele quesito para elevar o nível de transparência do portal de ECOS.

No trabalho de Souza et al. (2020), que se refere ao experimento exploratório realizado na etapa 1 da metodologia desta pesquisa, é apresentado um experimento com 
a ferramenta T2-UXT, com objetivo de auxiliar na avaliação das características que contribuem para a transparência em portais de ECOS. A T2-UXT monitora, de forma automatizada, a experiência do usuário (neste caso, desenvolvedor) no ambiente Web e gera artefatos (e.g., mapas de calor, mapas de rastreio do mouse e do olhar, entre outros) para documentá-la, o que permite a realização de análises e diagnósticos sobre o nível de transparência.

Os trabalhos de Fontão et al. (2017) e de Herbsleb et al. (2016) abordam fatores importantes em relação a DX e transparência em ECOS, respectivamente. No entanto, ainda não apresentam uma forma de como operacionalizar o monitoramento desses fatores ou sistematizar a avaliação de transparência atrelada a DX em ECOS, sendo esses pontos os diferenciais desta pesquisa.

A ideia é que o framework, que será desenvolvido nesta pesquisa, estenda e aprimore as definições do INTRASPE [Meireles et al. 2019] para cada uma das características de transparência (acessibilidade, usabilidade, informativo, entendimento e auditabilidade) a partir da DX em diferente portais de ECOS. Além disso, os experimentos que serão feitos com os desenvolvedores, com o auxílio da T2-UXT para monitorar a DX e gerar os artefatos, irão permitir a elaboração das heurísticas e/ou metaheurísticas de transparência.

\section{Contribuições Esperadas}

Esta pesquisa busca oferecer as seguintes contribuições tanto para a academia como para a indústria:

- Entendimento de como a transparência nos portais de ECOS afeta a DX;

- Mapeamentos sistemáticos de literatura sobre transparência em ECOS e sobre fatores de DX em ECOS;

- Conjunto de heurísticas e/ou metaheurísticas para transparência em portais de ECOS, elaboradas com base na DX;

- Criação de framework para avaliação de transparência em portais ECOS que possa ser utilizado por profissionais de organizações ou pesquisadores do campo;

- Evolução da ferramenta de captura multimodal T2-UXT à medida em que os experimentos forem realizados.

\section{Referências}

Amorim, S. S., Neto, F. S. S., McGregor, J. D., Almeida, E. S., e Chavez, C. F. G. (2017). How has the health of software ecosystems been evaluated?: A systematic review. In SBES'17: Proceedings of the 31st Brazilian Symposium on Software Engineering, pages 14-23.

Bader, F., Schön, E.-M., e Thomaschewski, J. (2017). Heuristics considering ux and quality criteria for heuristics. International Journal of Interactive Multimedia and Artificial Intelligence, 4:48-53.

Cappelli, C. (2009). Uma Abordagem Para Transparência Em Processos Organizacionais Utilizando Aspectos. Tese de doutorado, Pontifícia Universidade Católica do Rio de Janeiro, Rio de Janeiro, Brasil.

Cataldo, M. e Herbsleb, J. (2010). Architecting in software ecosystems: Interface translucence as an enabler for scalable collaboration. In ECSAW'10: Proceedings of the IV European Conference on Software Architecture Workshops, pages 65-72. 
Fagerholm, F. e Münch, J. (2012). Developer experience: Concept and definition. In 2012 International Conference on Software and System Process (ICSSP), pages 73-77.

Fonteyn, M., Kuipers, B., e Grobe, S. (1993). A description of think aloud method and protocol analysis. Qualitative Health Research - QUAL HEALTH RES, 3:430-441.

Fontão, A., Dias-Neto, A., e Viana, D. (2017). Investigating factors that influence developers' experience in mobile software ecosystems. In 2017 IEEE/ACM Joint 5th International Workshop on Software Engineering for Systems-of-Systems and 11th Workshop on Distributed Software Development, Software Ecosystems and Systems-of-Systems (JSOS), pages 55-58.

Fontão, A., Santos, R., e Dias-Neto, A. C. (2020). Devgo: Um modelo para governança de desenvolvedores em ecossistema de software móvel a partir de developer relations. In Anais Estendidos do XVI Simpósio Brasileiro de Sistemas de Informação, SBSI 2020, Porto Alegre, RS, Brasil. SBC - Sociedade Brasileira de Computação.

Hanssen, G. e Dybå, T. (2012). Theoretical foundations of software ecosystems. In Proceedings of the 4th International Workshop on Software Ecosystems (IWSECO) 3rd International Conference on Software Business (ICSOB), volume 879, pages 6-17.

Herbsleb, J., Kästner, C., e Bogart, C. (2016). Intelligently transparent software ecosystems. IEEE Software, 33(1):89-96.

Jansen, S., Brinkkemper, S., Finkelstein, A., e Bosch, J. (2009). Introduction to the proceedings of the first workshop on software ecosystems. In Proceedings of the First Workshop on Software Ecosystems, CEUR-WS, page 1-2.

Leite, J. C. S. P. e Cappelli, C. (2010). Software transparency. Business Information Systems Engineering, 2:127-139.

Lord, K. M. (2007). The Perils and Promise of Global Transparency: Why the Information. State University of New York Press, New York, USA.

Manikas, K. (2016). Revisiting software ecosystems research. Journal of Systems and Software, 117:84-103.

Meireles, A. I., Santos, R. P., e Cappelli, C. (2019). Um instrumento para avaliação e sugestões de mecanismos de transparência em portais de ecossistemas de software. iSys - Revista Brasileira de Sistemas de Informação, 12(6):05-38.

Quiñones, D., Rusu, C., e Rusu, V. (2018). A methodology to develop usability/user experience heuristics. Computer Standards Interfaces, 59:109-129.

Santos, R., Cappelli, C., Maciel, C., e Leite, J. C. S. P. (2016). Transparência em ecossistemas de software. In WDES'16: Anais do X Workshop em Desenvolvimento Distribuído de Software, Ecossistemas de Software e Sistemas-de-Sistemas, pages 75-79, Porto Alegre, RS, Brasil. SBC - Sociedade Brasileira de Computação.

Santos, R. P. (2016). Managing and Monitoring Software Ecosystem to Support Demand and Solution Analysis. Tese de doutorado, COPPE/UFRJ, Universidade Federal do Rio de Janeiro, Rio de Janeiro, Brasil.

Souza, K. E. S., Zacarias, R. O., Seruffo, M. C. R., e Santos, R. P. (2020). T2-uxt: A tool to support transparency evaluation in seco portals. In 34th Brazilian Symposium on Software Engineering (SBES '20), New York, NY, USA. ACM. 